\title{
Trends in Epidemiology and Management of Breast Cancer in Women Under 46 Years: Institutional Experience from a Tertiary Cancer Centre in Eastern India
}

\author{
Sourav Kumar Ghosh ${ }^{1}$, Sanskriti Poddar ${ }^{2}$, Krishnangshu Bhanja Choudhury ${ }^{3}$ \\ ${ }^{1}$ R.M.O. cum Clinical Tutor, Department of Radiotherapy, R. G. Kar Medical College \& Hospital, Kolkata, \\ India. \\ ${ }^{2}$ MD Post Graduate Trainee, Department of Radiotherapy, R. G. Kar Medical College \& Hospital, Kolkata, \\ India. \\ ${ }^{3}$ Assistant Professor, Department of Radiotherapy, R. G. Kar Medical College \& Hospital, Kolkata, India.
}

Corresponding Author: Krishnangshu Bhanja Choudhury

\begin{abstract}
Background: Breast cancer in younger women is a growing burden both in developed and Asian subcontinent. Despite studies showing varying results about the impact of age on treatment outcome and suboptimal survival, very few robust Indian studies have thrown light on this biologically different entity.
\end{abstract}

Methods: Histologically / cytologically confirmed cases of non-sarcomatous, female ductal breast carcinoma patients of age group less than and equal to 45 years of all stages attending radiotherapy department of R.G Kar Medical College between January 2016December 2018 were included in the study. Relevant information was obtained from patient's files/case records. Database was locked on $31^{\text {st }}$ March 2021.The baseline demographic profile, cancer subsites along with treatment provided were analysed using SPSS version 16 (IBM Inc, Armonk, New York, U.S.). Descriptive data are provided.

Results: Total 272 patients were eligible for the study as per the inclusion criteria with median age of 39 years (22-45 years). Majority were urban married Hindu females. Majority were locally advanced and node positive high grade disease as per AJCC $7^{\text {th }}$ staging system. Modified radical mastectomy was significantly higher than breast conservation surgery as the surgical modality (76 vs. $8.9 \%$ ). $31.2 \%, 54.5 \%$ patients received neoadjuvant and adjuvant chemotherapy respectively.61\% patients received curative intent radiotherapy either in conventional or hypofractionated schedule. Myelosuppression and oral mucositis were the major treatment related adverse events. Overall median PFS was 48 months.

Conclusion: Breast cancer in younger age group is distinct in terms of disease biology. Effective screening and diagnostics modalities with focus on mass awareness amongst patients and health care workers are the cornerstone of improving outcome and survival.

Key Words: breast cancer, young females, retrospective single institutional study

\section{INTRODUCTION}

Invasive breast cancer in females is a growing health concern both in developed and developing countries across the world in terms of incidence and mortality. According to Globocan 2020 statistics around 2.2 million new cases of breast cancer have been diagnosed worldwide in 2020 making it the commonest malignancy in females ahead of lung and cervical malignancies with an estimated cancer death rate of $6.9 \%$ amongst all cancers ${ }^{[1]}$. Breast cancer is the leading cause $(26.3 \%)$ of total cancer burden in Indian females with significant mortality rate ${ }^{[2]}$. Classically more common in postmenopausal women, there has been an age shift globally affecting a significant 
proportion of women in their $30 \mathrm{~s}$ and $40 \mathrm{~s}$. According to SEER approximately $5.6 \%$ of all invasive breast cancers occur in adolescents and young adults in US with some Asian countries even showing a higher propensity of young age group predilection compared to the western world due to multifactorial etiologies ${ }^{[3]}$. Studies from the central and northern part of our country have shown about $8 \%$ incidence of breast cancer in women below 35 years of age which is bit higher in comparison to the developed countries ${ }^{[4]}$. However very few studies are available from eastern India which has looked at the epidemiological trends and variations of young adult women with breast cancer which demands more research on this alarming entity.

Higher grade tumours, less ER expression, Her 2 overexpression, advanced stage of presentation, higher node positive disease has shown that breast cancer in earlier age of onset is certainly biologically aggressive and difficult to tackle ${ }^{[5]}$. Over the decades there has been a long debate whether age as an independent prognostic factor with studies showing varying results across the globe. Pregnancy, lactation, fertility issues, lack of awareness have always been the additional crucial variables in the management that have puzzled the clinicians more in compared to postmenopausal elderly cohort ${ }^{[6]}$. Malvia et al. in their recent study showed that $5 \%$ of breast cancer cases in India have inherited familial factors and approximately $2.9 \%$ of early onset breast cancer patients in India were found to have BRCA1/2 mutations ${ }^{[7]}$. Role of alcohol intake and obesity as risk factors in young adults are also less clear in contrast to the post-menopausal group that makes it a unique biological entity ${ }^{[8]}$. Data of breast cancer among young population especially from eastern part of India is lacking and our retrospective study is an attempt to identify the demographic profile, treatment and survival parameters in this population.

\section{METHODS}

Epidemiological data, treatment particulars and survival analysis were done for histologically/cytologically confirmed cases of non-sarcomatous, female ductal breast carcinoma patients of age group less than and equal to 45 years of all stages attending radiotherapy department of R.G Kar Medical College between January 2016-December 2018 in retrospective manner. Relevant information obtained from patient's files/case records. Database was locked on $31^{\text {st }}$ March 2021. Chi-square test for categorical variables and t test for non-categorical variables were used for comparisons. SPSS version 21(IBM Inc. Armonk, New York, USA) used for statistical derivation ${ }^{[10]}$. CTCAE v 4.03 scale was used for toxicity analysis ${ }^{[11]}$. For Calculating PFS (progression free survival) Kaplan-Maier survival plot was used ${ }^{[12]}$. PFS was defined as the time from date of diagnosis to tumour progression (local/regional/systemic). Median follow up time was calculated using reverse Kaplan Meier analysis. $\mathrm{P}$ value less than or equal to 0.05 was considered to be significant.

\section{RESULTS}

Total 272 patients were eligible for the study as per the inclusion criteria with median age of 39 years (22-45 years). The median follow up was 24 months for entire study population using reverse Kaplan Meier survival technique. Majority of the population were married urban females with good performance status and without significant family history as shown in table no 1.

Majority of the patients $(50.9 \%)$ presented with stage 3 disease as per AJCC $7^{\text {th }}$ staging system with $17.1 \%$ of the patients being upfront metastatic (bone mets most common). Nodal positivity rate was comparable across 3 years and was about 4 times than node negative disease as depicted in table no 2. 
Sourav Kumar Ghosh et.al. Trends in epidemiology and management of breast cancer in women under 46 years: Institutional experience from a tertiary cancer centre in Eastern India.

\begin{tabular}{|c|c|c|c|c|c|c|c|c|}
\hline \multicolumn{9}{|c|}{ Table: 1 Demographic parameters of the study population } \\
\hline \multirow{3}{*}{\multicolumn{2}{|c|}{ Parameters }} & \multicolumn{6}{|c|}{ Year } & \multirow[t]{3}{*}{ P VALUE } \\
\hline & & \multicolumn{2}{|c|}{$2016(\mathrm{~N}=77)$} & \multicolumn{2}{|c|}{$2017(\mathrm{~N}=91)$} & \multicolumn{2}{|c|}{$2018(\mathrm{~N}=104)$} & \\
\hline & & Count & Column N\% & Count & Column N \% & Count & Column N \% & \\
\hline \multirow[t]{5}{*}{ Age in years } & Mean & \multicolumn{2}{|l|}{38.48} & \multicolumn{2}{|l|}{37.68} & \multicolumn{2}{|l|}{38.08} & \multirow[t]{5}{*}{0.593} \\
\hline & Median & \multicolumn{2}{|l|}{40.00} & \multicolumn{2}{|l|}{39.00} & \multicolumn{2}{|l|}{39.00} & \\
\hline & S.D & \multicolumn{2}{|l|}{5.330} & \multicolumn{2}{|l|}{5.140} & \multicolumn{2}{|l|}{4.737} & \\
\hline & Minimum & \multicolumn{2}{|l|}{22} & \multicolumn{2}{|l|}{27} & \multicolumn{2}{|l|}{25} & \\
\hline & Махітит & \multicolumn{2}{|l|}{45} & \multicolumn{2}{|l|}{45} & \multicolumn{2}{|l|}{45} & \\
\hline \multirow{5}{*}{ Menarche in years } & Mean & \multicolumn{2}{|l|}{13.45} & \multicolumn{2}{|l|}{13.68} & \multicolumn{2}{|l|}{13.77} & \multirow[t]{5}{*}{0.387} \\
\hline & Median & & & 14.00 & & 14.00 & & \\
\hline & S.D & 1.518 & & 1.673 & & 1.429 & & \\
\hline & Minimum & 10 & & 10 & & 11 & & \\
\hline & Mахітит & 17 & & 17 & & 17 & & \\
\hline ECOG Performance Status & 1 & 53 & $68.8 \%$ & 60 & $65.9 \%$ & 64 & $61.5 \%$ & 0.594 \\
\hline & 2 & 15 & $19.5 \%$ & 24 & $26.4 \%$ & 24 & $23.1 \%$ & \\
\hline & 3 & 8 & $10.4 \%$ & 6 & $6.6 \%$ & 12 & $11.5 \%$ & \\
\hline & 4 & 1 & $1.3 \%$ & 1 & $1.1 \%$ & 4 & $3.8 \%$ & \\
\hline Residence & Urban & 40 & $51.9 \%$ & 45 & $49.5 \%$ & 59 & $56.7 \%$ & 0.584 \\
\hline & Rural & 37 & $48.1 \%$ & 46 & $50.5 \%$ & 45 & $43.3 \%$ & \\
\hline Martial_status & Married & 71 & $92.2 \%$ & 85 & $93.4 \%$ & 94 & $90.4 \%$ & 0.738 \\
\hline & Unmarried & 6 & $7.8 \%$ & 6 & $6.6 \%$ & 10 & $9.6 \%$ & \\
\hline Parity & Multipara & 62 & $80.5 \%$ & 75 & $82.4 \%$ & 79 & $76.0 \%$ & 0.517 \\
\hline & Nulliparous & 15 & $19.5 \%$ & 16 & $17.6 \%$ & 25 & $24.0 \%$ & \\
\hline OCP & Yes & 28 & $36.4 \%$ & 33 & $36.3 \%$ & 39 & $37.5 \%$ & 0.981 \\
\hline & No & 49 & $63.6 \%$ & 58 & $63.7 \%$ & 65 & $62.5 \%$ & \\
\hline Family history & 0 & 77 & $100.0 \%$ & 88 & $96.7 \%$ & 101 & $97.1 \%$ & 0.292 \\
\hline & 1 & 0 & $0.0 \%$ & 3 & $3.3 \%$ & 3 & $2.9 \%$ & \\
\hline Second Malignancy & 0 & 77 & $100.0 \%$ & 91 & $100.0 \%$ & 104 & $100.0 \%$ & NA \\
\hline
\end{tabular}

\begin{tabular}{|c|c|c|c|c|c|c|c|c|}
\hline \multirow{3}{*}{\multicolumn{2}{|c|}{ TNM PARAMETERS }} & \multicolumn{6}{|l|}{ Year } & \multirow[t]{3}{*}{ P VALUE } \\
\hline & & \multicolumn{2}{|l|}{2016} & \multicolumn{2}{|l|}{2017} & \multicolumn{2}{|l|}{2018} & \\
\hline & & Count & Column N \% & Count & Column N \% & Count & Column N \% & \\
\hline \multirow[t]{6}{*}{$T$} & T1 & 4 & $5.2 \%$ & 8 & $8.8 \%$ & 4 & $3.8 \%$ & \multirow[t]{6}{*}{0.880} \\
\hline & $\mathrm{T} 2$ & 29 & $37.7 \%$ & 36 & $39.6 \%$ & 36 & $34.6 \%$ & \\
\hline & T3 & 24 & $31.2 \%$ & 22 & $24.2 \%$ & 37 & $35.6 \%$ & \\
\hline & T4B & 10 & $13.0 \%$ & 13 & $14.3 \%$ & 15 & $14.4 \%$ & \\
\hline & $\mathrm{T} 4 \mathrm{C}$ & 9 & $11.7 \%$ & 10 & $11.0 \%$ & 11 & $10.6 \%$ & \\
\hline & T4D & 1 & $1.3 \%$ & 2 & $2.2 \%$ & 1 & $1.0 \%$ & \\
\hline \multirow[t]{4}{*}{$\mathbf{N}$} & N0 & 15 & $19.5 \%$ & 20 & $22.0 \%$ & 23 & $22.1 \%$ & \multirow[t]{4}{*}{0.994} \\
\hline & N1 & 29 & $37.7 \%$ & 31 & $34.1 \%$ & 38 & $36.5 \%$ & \\
\hline & $\mathrm{N} 2$ & 17 & $22.1 \%$ & 22 & $24.2 \%$ & 21 & $20.2 \%$ & \\
\hline & N3 & 16 & $20.8 \%$ & 18 & $19.8 \%$ & 22 & $21.2 \%$ & \\
\hline \multirow[t]{2}{*}{$\mathbf{M}$} & M1 & 12 & $15.6 \%$ & 15 & $16.5 \%$ & 20 & $19.2 \%$ & \multirow[t]{2}{*}{0.790} \\
\hline & $\mathrm{MO}$ & 65 & $84.4 \%$ & 76 & $83.5 \%$ & 84 & $80.8 \%$ & \\
\hline \multirow[t]{7}{*}{ TNM stage* } & $1 \mathrm{~A}$ & 2 & $2.6 \%$ & 4 & $4.4 \%$ & 2 & $1.9 \%$ & \multirow[t]{7}{*}{0.996} \\
\hline & $2 \mathrm{~A}$ & 8 & $10.4 \%$ & 11 & $12.1 \%$ & 11 & $10.6 \%$ & \\
\hline & $2 \mathrm{~B}$ & 14 & $18.2 \%$ & 17 & $18.7 \%$ & 18 & $17.3 \%$ & \\
\hline & $3 \mathrm{~A}$ & 18 & $23.4 \%$ & 19 & $20.9 \%$ & 20 & $19.2 \%$ & \\
\hline & $3 \mathrm{~B}$ & 10 & $13.0 \%$ & 13 & $14.3 \%$ & 14 & $13.5 \%$ & \\
\hline & $3 \mathrm{C}$ & 13 & $16.9 \%$ & 12 & $13.2 \%$ & 19 & $18.3 \%$ & \\
\hline & 4 & 12 & $15.6 \%$ & 15 & $16.5 \%$ & 20 & $19.2 \%$ & \\
\hline
\end{tabular}

$32 \%, 30 \%$ and $25 \%$ cases were the proportion of triple negative disease from 2016-18 respectively with $40 \%$ presenting with grade 3 disease as per BloomRichardson grading system(table no 3 ). Her 2 positivity rate showed a rising trend as years progressed with more patients proceeding to costlier tests like FISH for Her-2-neu score 2 in IHC.

As for treatment a total of 85 patients $(31.2 \%)$ received neo-adjuvant chemotherapy, median number of cycles was 4(3-6) with TAC (Docetaxel, Doxorubicin, Cyclophosphamide) being the most common regimen used followed by FEC (5 FU, Epirubicin, Cyclophosphamide). An average of $54.5 \%$ patients received adjuvant chemotherapy with TAC being the most common regimen used. Modified radical mastectomy was by far the commonest surgical approach in comparison to breast conservation surgery in our study 
Sourav Kumar Ghosh et.al. Trends in epidemiology and management of breast cancer in women under 46 years: Institutional experience from a tertiary cancer centre in Eastern India.

population (76 vs. $8.9 \%)$. $61 \%$ of the total population received curative intent radiation therapy with conventional fractionation (50 Gy $/ 25$ fractions) being the most used fractionation schedule $(33.3 \%)$. $17.2 \%$ patients received palliative chemotherapy for metastatic disease. $8.4 \%$ of total study population received palliative radiotherapy for bone metastasis with $76.9 \%$ of all bone mets patients receiving oral or intravenous bisphosphonates (oral Ibandronate / i.v Zoledronic acid)(table 4). $41 . \%$ and $7.7 \%$ were treated with Tamoxifen and aromatase inhibitor respectively for hormone positive disease. $33 \%$ of patients of all Her 2 positive disease received desired dose of Trastuzumab as per protocol(table 5).

\begin{tabular}{|c|c|c|c|c|c|c|c|c|}
\hline \multicolumn{9}{|c|}{ Table 3. Tumour characteristics } \\
\hline \multirow{3}{*}{\multicolumn{2}{|c|}{ Parameters }} & \multicolumn{6}{|c|}{ - } & \multirow{3}{*}{$\mathrm{P}$ value } \\
\hline & & \multicolumn{2}{|l|}{2016} & \multicolumn{2}{|l|}{2017} & \multicolumn{2}{|l|}{2018} & \\
\hline & & Count & Column N \% & Count & Column N \% & Count & Column N \% & \\
\hline \multirow[t]{2}{*}{ ER } & NEG & 33 & $42.9 \%$ & 43 & $47.3 \%$ & 45 & $43.3 \%$ & \multirow{2}{*}{0.808} \\
\hline & POS & 44 & $57.1 \%$ & 48 & $52.7 \%$ & 59 & $56.7 \%$ & \\
\hline \multirow[t]{2}{*}{ PR } & NEG & 47 & $61.0 \%$ & 54 & $59.3 \%$ & 61 & $58.7 \%$ & \multirow{2}{*}{0.948} \\
\hline & POS & 30 & $39.0 \%$ & 37 & $40.7 \%$ & 43 & $41.3 \%$ & \\
\hline \multirow[t]{3}{*}{ HER 2 neu } & EQUI & 1 & $1.3 \%$ & 4 & $4.4 \%$ & 3 & $2.9 \%$ & \multirow{3}{*}{0.415} \\
\hline & NEG & 60 & $77.9 \%$ & 64 & $70.3 \%$ & 69 & $66.3 \%$ & \\
\hline & POS & 16 & $20.8 \%$ & 23 & $25.3 \%$ & 32 & $30.8 \%$ & \\
\hline \multirow[t]{3}{*}{ Grade } & 1 & 12 & $15.6 \%$ & 14 & $15.4 \%$ & 16 & $15.4 \%$ & \multirow{3}{*}{0.995} \\
\hline & 2 & 34 & $44.2 \%$ & 42 & $46.2 \%$ & 45 & $43.3 \%$ & \\
\hline & 3 & 31 & $40.3 \%$ & 35 & $38.5 \%$ & 43 & $41.3 \%$ & \\
\hline \multirow[t]{3}{*}{ Margin } & $0 * *$ & 5 & $6.5 \%$ & 6 & $6.6 \%$ & 8 & $7.7 \%$ & \multirow{3}{*}{0.973} \\
\hline & NEG & 52 & $67.5 \%$ & 62 & $68.1 \%$ & 73 & $70.2 \%$ & \\
\hline & POS & 20 & $26.0 \%$ & 23 & $25.3 \%$ & 23 & $22.1 \%$ & \\
\hline \multirow[t]{2}{*}{ LVSI } & NEG & 45 & $58.4 \%$ & 47 & $51.6 \%$ & 59 & $56.7 \%$ & \multirow{2}{*}{0.644} \\
\hline & POS & 32 & $41.6 \%$ & 44 & $48.4 \%$ & 45 & $43.3 \%$ & \\
\hline \multirow[t]{2}{*}{ Site } & Left & 43 & $55.8 \%$ & 50 & $54.9 \%$ & 57 & $54.8 \%$ & \multirow{2}{*}{0.989} \\
\hline & Right & 34 & $44.2 \%$ & 41 & $45.1 \%$ & 47 & $45.2 \%$ & \\
\hline \multirow[t]{5}{*}{ Quadrant } & Upper Outer & 45 & $58.4 \%$ & 43 & $47.3 \%$ & 51 & $49.0 \%$ & \multirow{5}{*}{0.236} \\
\hline & Lower Outer & 14 & $18.2 \%$ & 25 & $27.5 \%$ & 26 & $25.0 \%$ & \\
\hline & Lower Inner & 9 & $11.7 \%$ & 12 & $13.2 \%$ & 12 & $11.5 \%$ & \\
\hline & Upper Inner & 4 & $5.2 \%$ & 11 & $12.1 \%$ & 10 & $9.6 \%$ & \\
\hline & Central / NAC & 5 & $6.5 \%$ & 0 & $0.0 \%$ & 5 & $4.8 \%$ & \\
\hline
\end{tabular}

\begin{tabular}{|l|l|l|l|l|l|l|l|}
\hline \multicolumn{7}{|c|}{ Table 4. Adjuvant chemotherapy in non-metastatic patients } & \multirow{2}{*}{ P VALUE } \\
\hline \multirow{3}{*}{ ACT DRUGS } & Year & 2016 & 2017 & 2018 & \\
\cline { 2 - 8 } & Count & Column N \% & Count & Column N \% & Count & Column N \% & \\
\hline No adjuvant & 30 & $46.2 \%$ & 34 & $44.7 \%$ & 38 & $45.2 \%$ & \multirow{2}{*}{0.972} \\
\hline AC & 2 & $3.1 \%$ & 2 & $2.6 \%$ & 4 & $4.8 \%$ & \\
\hline AC,T & 3 & $4.6 \%$ & 4 & $5.3 \%$ & 2 & $2.4 \%$ \\
\hline FAC & 3 & $4.6 \%$ & 4 & $5.3 \%$ & 2 & $2.4 \%$ \\
\hline FAC,T & 1 & $1.5 \%$ & 1 & $1.3 \%$ & 2 & $2.4 \%$ \\
\hline FEC & 2 & $3.1 \%$ & 1 & $1.3 \%$ & 3 & $3.6 \%$ \\
\hline FEC,T & 2 & $3.1 \%$ & 1 & $1.3 \%$ & 1 & $1.2 \%$ \\
\hline GEM,DOCE & 0 & $.0 \%$ & 1 & $1.3 \%$ & 1 & $1.2 \%$ \\
\hline T & 8 & $12.3 \%$ & 15 & $19.7 \%$ & 10 & $11.9 \%$ \\
\hline TAC & 14 & $21.5 \%$ & 13 & $17.1 \%$ & 21 & $25.0 \%$ & \\
\hline Doxorubicin, C-Cyclophosphamide, T/ Doce - Docetaxel, F-5Fluorouracil, E- Epirubicin, Gem - Gemcitabine \\
\hline
\end{tabular}

\begin{tabular}{|c|c|c|c|c|c|c|c|c|c|}
\hline & & Table & Horm & nal therapy $\&$ & rrgeted & erapy & & & \\
\hline Treatment & & & Year & & & & & & $\mathrm{P}$ value \\
\hline & & & & & & & & & \\
\hline & & & Count & Column N\% & Count & Column N \% & Count & Column N\% & \\
\hline Hormone Therapy & Anastrazole & & 0 & $.0 \%$ & 0 & $.0 \%$ & 1 & $1.0 \%$ & 0.312 \\
\hline & Letrozole & & 2 & $2.6 \%$ & 2 & $2.2 \%$ & 2 & $1.9 \%$ & \\
\hline & NO hormone therapy & & 49 & $63.6 \%$ & 48 & $52.7 \%$ & 51 & $49.0 \%$ & \\
\hline & Tamoxifen (TMX) & & 24 & $31.2 \%$ & 40 & $44.0 \%$ & 50 & $48.1 \%$ & \\
\hline & TMX, Anastrazole & & 2 & $2.6 \%$ & 1 & $1.1 \%$ & 0 & $.0 \%$ & \\
\hline HER 2 neu & TRASTUZUMAB & Yes & 6 & $37.5 \%$ & 8 & $34.8 \%$ & 8 & $25.0 \%$ & 0.604 \\
\hline positive & & No & 10 & $62.5 \%$ & 15 & $65.2 \%$ & 24 & $75.0 \%$ & \\
\hline
\end{tabular}


Sourav Kumar Ghosh et.al. Trends in epidemiology and management of breast cancer in women under 46 years: Institutional experience from a tertiary cancer centre in Eastern India.

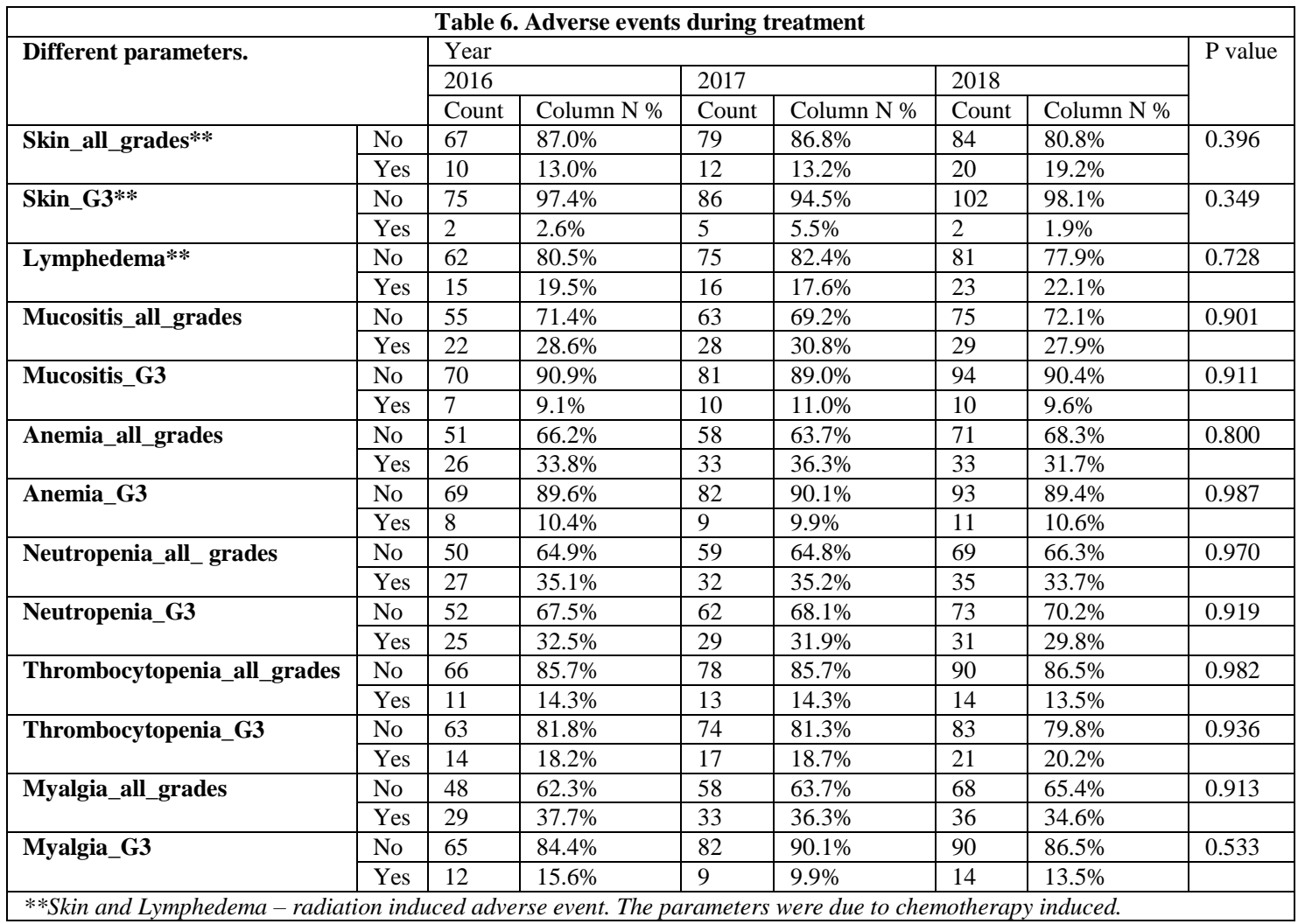

\begin{tabular}{|l|l|l|l|l|}
\hline Table 7a. Case Processing Summary for Progression Free Survival analysis. \\
\hline Year & Total N & N of Events & Censored \\
\cline { 4 - 5 } & & & N & Percent \\
\hline $\mathbf{2 0 1 6}$ & 77 & 19 & 58 & $75.3 \%$ \\
\hline $\mathbf{2 0 1 7}$ & 91 & 25 & 66 & $72.5 \%$ \\
\hline $\mathbf{2 0 1 8}$ & 104 & 25 & 79 & $76.0 \%$ \\
\hline Overall & 272 & 69 & 203 & $74.6 \%$ \\
\hline
\end{tabular}

\begin{tabular}{|c|c|c|c|c|c|c|c|c|}
\hline \multirow{3}{*}{ Year } & \multicolumn{4}{|l|}{ Mean $^{a}$} & \multicolumn{4}{|l|}{ Median } \\
\hline & \multirow{2}{*}{ Estimate } & \multirow[t]{2}{*}{ Std. Error } & \multicolumn{2}{|c|}{ 95\% Confidence Interval } & \multirow{2}{*}{ Estimate } & \multirow[t]{2}{*}{ Std. Error } & \multicolumn{2}{|c|}{ 95\% Confidence Interval } \\
\hline & & & Lower Bound & Upper Bound & & & Lower Bound & Upper Bound \\
\hline 2016 & 46.754 & 2.656 & 41.549 & 51.959 & 57.000 & 6.537 & 44.188 & 69.812 \\
\hline 2017 & 39.740 & 1.656 & 36.495 & 42.985 & 46.000 & 1.512 & 43.037 & 48.963 \\
\hline 2018 & 29.499 & 1.185 & 27.175 & 31.822 & 36.000 & 4.183 & 27.802 & 44.198 \\
\hline Overall & 42.931 & 1.583 & 39.829 & 46.034 & 48.000 & 2.763 & 42.584 & 53.416 \\
\hline
\end{tabular}

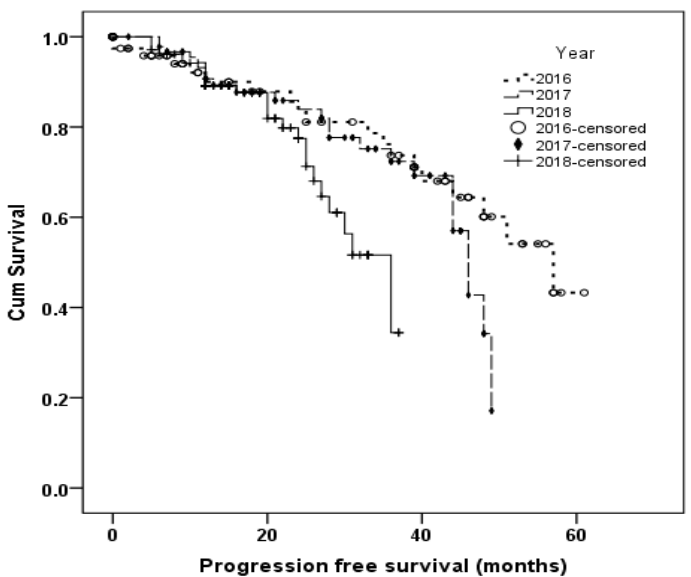

Figure 1. Progression free survival analysis comparison among patients according to year wise recruitment with Log Rank test - Chi Square 6.401, df 2, p value 0.041.
Amongst all, $31.4 \%$ and $19 \%$ patients developed grade 3 neutropenia and thrombocytopenia respectively during treatment. Around $10 \%$ of the patients developed grade 3 oral mucositis during chemotherapy/radiation. Skin toxicities, lymphedema of the arm are the different non haematological toxicities encountered during and after the treatment as detailed below in table no. 6 .

Total 69 patients $(25.3 \%)$ experienced an event either in form of local/regional or systemic progression or death. The Progression Free Survival were 57, 46 and 36 months respectively for 
patients recruited in 2016, 2017 and 2018, with significant log rank test, $\mathrm{p}$ value 0.041 . (table 7 and figure 1). Overall median progression free survival for the study population was 48 months as shown in the KM survival plot.

\section{DISCUSSION}

Breast cancer in females has shown a clear age shift both in developed and Asian population in past few years posing a threat to younger adults. Earlier studies published from different parts of our country showed incidence of 5-8\% of breast cancer cases in women $<35$ years age group with a mean age of diagnosis ranging from 45-50 years. Sofi $\mathrm{N}$ et.al in their study from central India showed approximately $49 \%$ of the cases being diagnosed under 45 years of age ${ }^{[9]}$. Thangjam et.al.in their study from Manipur showed that $31 \%$ of all breast cancer cases were diagnosed below 40 years of age which is more or less in line with our study where $30.3 \%$ of all breast cases were diagnosed in less than 45 years age group [10]. However it is somewhat lower in percentage to other available evidences may be due to more attrition of patients owing to several socioeconomic factors ${ }^{[11]}$.

As similar to previous data, breast cancer in our study was commoner in urban females $(52.7 \%)$ as compared to rural though not statistically significant ${ }^{[12]}$. Disease was more common in married multiparous women which may be linked to earlier age of marriage and lack of proper contraception practices $(36.7 \%$ users of oral contraceptive pills).

Stage III infiltrating ductal carcinoma was the commonest presenting stage in our study which resembles earlier published studies like Das et.al ${ }^{[13]}$. Nodal positivity rate in our patients were higher than some earlier published studies based on young age breast malignancy may be due to late stage of presentation due to lack of health awareness and education [14]. Percentage of patients presenting with triple negative disease and high grade tumours was comparable with some other Indian studies published earlier ${ }^{[15]}$.

There was a striking difference between modified radical mastectomy and breast conservation surgery found in our studies that was in contrast to most international and even some national studies which reflected the need for more focus on breast cancer screening to pick up early operable and large operable disease as well as to popularize the practice of doing BCS in our setting ${ }^{[16]}$.

Regarding adherence to treatment regarding chemotherapy, radiation therapy, targeted therapy the findings in our study reflected the real time situation due to logistics issue and multiple factors like distance from treatment centre, poverty, lack of health knowledge amongst patients and caregivers which was in contrast with the western world data that demonstrated better compliance and survival ${ }^{[17]}$.

Regarding survival data, $74.7 \%$ of patients were disease free in our study till last follow up which was more or less similar to the results found from Saudi Arabia and higher as compared to Egyptian data on young women having breast cancer [18]. However with varying patient characteristics, sample size and follow up period wide range of PFS data have been found in different studies that makes any direct comparison difficult.

We accept our study had its limitations in form of being single institutional, having low sample size, more attrition and being retrospective. However large prospective studies on this topic are needed for further knowledge and intervention.

\section{CONCLUSION}

Breast cancer in younger women is a distinct and growing entity which calls for effective screening programme and timely diagnosis along with improved health care resource and practices to optimize the outcome.

Acknowledgement: None 

years: Institutional experience from a tertiary cancer centre in Eastern India.

Conflict of Interest: None

\section{Source of Funding: None}

Ethical Approval: Approved

\section{REFERENCES}

1. Sung H, Ferlay J, Siegel RL, Laversanne M, Soerjomataram I, Jemal A, Bray F. Global Cancer Statistics 2020: GLOBOCAN Estimates of Incidence and Mortality Worldwide for 36 Cancers in 185 Countries. CA Cancer J Clin. 2021;71(3):209-249.

2. Shetty R, Mathew RT, Vijayakumar M. Incidence and pattern of distribution of cancer in India: A secondary data analysis from six population-.based cancer registries. Cancer Res Stat Treat 2020; 3:678-82.

3. Johnson, R. H., Anders, C. K., Litton, J. K., Ruddy, K. J., \& Bleyer, A. Breast cancer in adolescents and young adults. Pediatric blood \& cancer 2018; 65(12), e27397.

4. Raina V, Bhutani M, Bedi R, Sharma A, Deo SV, Shukla NK, Mohanti BK, Rath GK. Clinical features and prognostic factors of early breast cancer at a major cancer center in North India. Indian $\mathbf{J}$ Cancer 2005; 42:40-5.

5. Anders CK, Johnson R, Litton J, Phillips $\mathrm{M}$, Bleyer A. Breast cancer before age 40 years. Semin Oncol. 2009; 36(3):237-49.

6. Zhang X, Yang J, Cai H, Ye Y. Young age is an independent adverse prognostic factor in early stage breast cancer: a population-based study. Cancer Manag Res. 2018;10:4005-18.

7. Malvia S, Bagadi SA, Dubey US, Saxena S. Epidemiology of breast cancer in Indian women. Asia Pac J Clin Oncol. 2017;13(4):289-295.

8. Shin A, Sandin S, Lof M, et al. Alcohol consumption, body mass index and breast cancer risk by hormone receptor status: Women' Lifestyle and Health Study. BMC Cancer. 2015;15:881.
9. Sofi NY, Jain M, Kapil U, Yadav CP. Epidemiological characteristics of breast cancer patients attending a tertiary health-care institute in the National Capital Territory of India. J Can Res Ther 2019;15:1087-91.

10. Thangjam S, Laishram RS, Debnath K. Breast carcinoma in young females below the age of 40 years: A histopathological perspective. South Asian J Cancer. 2014;3(2):97-100.

11. Chopra B, Kaur V, Singh K, Verma M, Singh S, Singh A. Age shift: Breast cancer is occurring in younger age groups - Is it true?. Clin Cancer Investig J 2014;3:526-9.

12. Thakur J S, Budukh A, Kapoor R, Malhotra P, Bashar MA, Kathirvel S, Dixit R, Arora P, Bhatia S, Badwe R A. Urban-rural differences in cancer incidence and pattern in Punjab and Chandigarh: Findings from four new population-based cancer registries in North India. Int $\mathrm{J}$ Non-Commun Dis 2017;2:49-55.

13. Das U, Lakshmaiah KC, Lokanatha D, Babu GK, Jacob L, Babu S. Breast cancer in women of younger than 35 years: A single center study. J Mol Biomark Diagn 2015; 6(6):1.

14. Walker RA, Lees E, Webb MB, Dearing SJ. Breast carcinomas occurring in young women $(<35$ years $)$ are different. Br J Cancer. 1996;74(11):1796-1800.

15. Ghosh J, Gupta S, Desai S, Shet T, Radhakrishnan S, Suryavanshi P, et al. Estrogen, progesterone and HER2 receptor expression in breast tumors of patients, and their usage of HER2targeted therapy, in a tertiary care centre in India. Indian J Cancer 2011;48:391-6.

16. Sinnadurai S, Kwong A, Hartman M, et al. Breast-conserving surgery versus mastectomy in young women with breast cancer in Asian settings. BJS Open. 2018;3(1):48-55.

17. Cowppli-Bony A, Trétarre B, Marrer E, Defossez G, Daubisse-Marliac L, Coureau G, et al. Compliance with clinical guidelines for breast cancer 
Sourav Kumar Ghosh et.al. Trends in epidemiology and management of breast cancer in women under 46 years: Institutional experience from a tertiary cancer centre in Eastern India.

management: A population-based study of quality-of-care indicators in France. PLoS ONE 2019;14(10): e0224275.

18. Elkum N, Dermime S, Ajarim D, AlZahrani A, Alsayed A, Tulbah A, Al Malik O, Alshabanah M, Ezzat A, AlTweigeri $\mathrm{T}$. Being 40 or younger is an independent risk factor for relapse in operable breast cancer patients: the Saudi Arabia experience. BMC cancer. 2007;7(1):1-8.
How to cite this article: Ghosh SK, Poddar $\mathrm{S}$, Choudhury KB. Trends in epidemiology and management of breast cancer in women under 46 years: Institutional experience from a tertiary cancer centre in Eastern India. International Journal of Research and Review. 2021; 8(7): 278-285. DOI: https://doi.org/10.52403/ijrr.20210739 